\title{
On the Scalability Problem of Highway Ad hoc Network
}

\author{
Florent Kaisser \\ IEF \\ Université Paris-Sud \\ Orsay, France \\ Email: florent.kaisser@u-psud.fr
}

\author{
Véronique Vèque \\ IEF \\ Université Paris-Sud \\ Orsay, France \\ Email: veronique.veque@u-psud.fr
}

\begin{abstract}
Vehicular Ad hoc Network in a highway is composed of high speed vehicles or nodes which induce fast topology changes in their configuration. In order to solve the connectivity and scalability problems of VANETs, we introduce the architecture of a Vehicular hybrid ad hoc network (VHANET). Using some routing protocol extensions, the VHANET allows ad hoc islands to be interconnected among each other using a infrastructure network. Our simulation results show that the performances, compared to a flat network, are greatly enhanced. In addition, we study a nearly optimal AP density for the use of our architecture.
\end{abstract}

\section{Vehicular Ad Hoc Networks and Routing}

Vehicular Network is a new capability for drivers to enhance safety on roads and then, to provide Internet access services. Vehicular Networks can be formed either by deployment of a telecommunication network infrastructure or by using $\mathrm{Ad}$ Hoc communications between vehicles. In the first case, some access points are distributed along the road, each one connected to other through a wired network allowing the vehicles to connect to the AP (Access Point). The main drawbacks of a complete road coverage are prohibitive costs and inefficient use in case of light traffic. On the other hand, a Vehicular Ad Hoc Network (VANET) is a special kind of Mobile Ad-Hoc Networks (MANET), where vehicles equipped with wireless devices constitute a network without any additional infrastructure.

In VANET [1], the vehicles act both as hosts and routers, being either packets source, destination or forwarder. VANET networks can be used for two kinds of applications: safety applications like alert diffusion, road foreseen, and user oriented applications like Internet access, VoIP, file transfers or advertising services. Both types of applications have different constraints in terms of delay, bandwidth and reliability. For user oriented applications, data rate will usually be greater than for safety applications. Safety applications are mainly interested with reliability and delay guarantee. Moreover, we consider safety and user-oriented applications which use unicast communications. For these unicast communications, a routing protocol is required. MANET routing protocols were designed to establish paths even with a dynamic network topology. With VANET, the difficulty comes from the nodes high mobility because the paths have to be more often established and a lot of signaling messages are broadcasted. The second problem comes from the convergence delay which has to be less than the topology change interval in order to achieve an accurate routing process.

For these main reasons, several studies have shown that it is not reasonable to use a proactive routing protocol for VANET where the vehicles speed implies a highly dynamic topology. In contrast, either reactive or geographical protocols are more robust to frequent topology changes. Particular routing techniques are thus studied for the VANET [1], [2] but other studies has demonstrated that DSR (Dynamic Source Routing, a reactive ad hoc protocol [3]) operates as well in a VANET [4].

To find a route, a reactive protocol broadcasts a RouteRequest message in the network. To avoid a network saturation, the hop number is limited to the maximum value of a Time To Live (TTL) parameter defined for the RouteRequest. When a network is very large, a node can not search the farthest nodes due to the limited value of TTL. It means that ad hoc reactive protocols are not truly scalable.

Geographic ad hoc routing protocols are also used for VANET. In a near future, most of the vehicles will be equipped with a positioning system such as GPS (Global Positioning System). VANET protocols can take advantage of geographical information like the destination position, avoiding thus path maintaining or routing table. But when a vehicle sends packets, the destination node position must be known. This is made with a location protocol which has to be efficient in order to preserve the inherent scalability of geographic routing protocols. The basic flooding-based approach [5] consists in broadcasting a position request through the entire network and generates a lot of signaling messages as each node receiving this request, forwards it until the destination node sends the response upon delivery. Obviously, this method is not scalable and produces a high overhead. Except flooding approach, another approach uses a rendez-vous mechanism. In rendezvous-based protocols, all nodes (potential senders or receivers) in the network agree upon a mapping that maps each nodes unique identifier to location servers. They will be the rendezvous nodes where periodical location updates will be stored and location queries will be looked up. As an example, GHLS (Geographic Hashing Location Service) protocol [5] uses location servers which register the position 


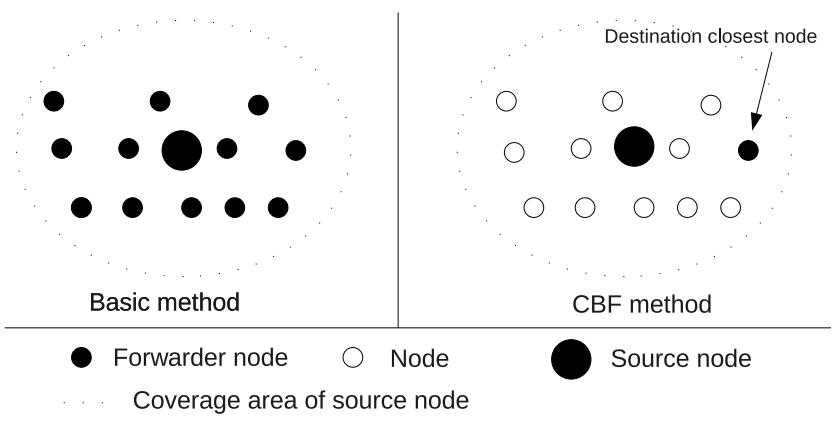

Fig. 1. CBF optimisation [9]

of several nodes. As soon as a node has moved, an update is processed: the new position is sent to its server. When a node is seeking another node position, it sends a request to the server which responds to it with the appropriated information. A hashing function is used to determine the server position. This function computes a geographic position with the ID destination node. The server is the closest node to this position. Thus, only the node between the sender and the server forwards the request and only the node between the destination and the server forwards the update message. As a consequence, the updating and searching overhead is reduced. Other scalable location protocols have been introduced: LAR (Location-Aided Routing) [6] , DREAM (A Distance Routing Effect Algorithm for Mobility) [7] or GLS (Grid Location Service) [8]. Sometimes, routing and location protocols are performed together, like in LAR or DREAM.

It is interesting to take advantage of our particular considered topology: MANET on a highway. With this line topology, a geographic routing protocol using the CBF (ContentionBased Forwarding) optimization [9] will operate at best. With a rendez-vous protocol and CBF improvement, only one node by area of coverage forwards the request (Fig. 1). Without this improvement, all nodes in the coverage area forward the request and the overhead increases with the vehicles density.

A common feature among geographic and reactive topological protocols is that they process the request (either to find a route or a position) as same, just before sending the data packets. However, reactive protocol does not allow a global optimization as we have seen for the geographic protocols with the optimized location protocol.

In ad hoc networking, a major problem is the scalability. Scalability and other performance aspects of ad hoc networking have been studied via simulation (e.g. [10], [11], [12]) or via theoretical analysis (e.g. [13], [14]).

In the case of a highway, we have a line topology and the scalability problem is not resolved neither with reactive protocols nor geographic protocols. At best, with a geographic protocol using the CBF improvement, the overhead is proportional to the highway length. In addition, the line topology of highway limits the spatial frequency reuse. Without spatial reuse possibility, a solution for scalability problem is a hybrid ad hoc network.
In this paper, we introduce the architecture of a Vehicular hybrid ad hoc network (VHANET). To manage both mobility and routing in VHANET, we use the reactive routing protocol DSR. Next section is dedicated to DSR extensions we are introducing to adapt it to a VHANET context and to overcome its scalability problems. In third section, we present simulation results to compare a flat network with our hybrid network.

\section{Vehicular Hybrid Ad hoc Network}

\section{A. Hybrid Ad Hoc Netwok}

As described above, geographic routing protocol has the same scalability impairment as the reactive routing protocol in a highway topology. We are interested here to extend a reactive ad hoc protocol for our VHANET architecture in order to make it more effective and scalable. Several extensions of reactive protocols have been proposed for the hybrid ad hoc network. $A O D V+[15]$ is an extension of AODV [16] for hybrid ad hoc network. This extension is an implementation for NS2 [17] of global connectivity for mobile ad hoc networks described in the IETF draft [18]. The first extension of DSR [3] for hybrid ad hoc network have been proposed in [19]. This extension provides to use several interfaces, i.e. wired and wireless, with DSR. A draft of IETF DYMO [20] has also been proposed a new version of DSR. In this draft, a similar method of the global connectivity for mobile ad hoc networks is developed to discover the closest gateway. However, all proposed protocols cited above are only dealing with Internet access and not, with true hybrid network. In the other hand, several vehicular hybrid ad hoc networks have been proposed (e.g. [21]), but the studies are also limited to Internet access. In our work, we are interesting in providing a total connectivity between the vehicles (i.e. node) using hybrid architecture and DSR extensions. Thus, our scalability proposals are based on both Vehicle-to-Vehicle (V2V) and Vehicle-to-Infrastructure (V2I) communications. After introducing the VHANET architecture, we will describe our reactive routing protocol extensions.

\section{B. Architecture of VHANET}

As stated in introduction, a pure ad hoc system may be fragmented into several ad hoc networks due to the clustering of cars, or due to the limited TTL. The Vehicular Hybrid ad hoc architecture is a way to overcome this connectivity problem. From a monetary point of view, this architecture allows to cover wide area with low nodes density and provides higher throughput than present cellular network like a UMTS network.

In such architecture, the mobile nodes can communicate with each other, like in ad hoc communications, or with an Access Point (AP). As APs are also connected together, we talk about an infrastructure network or backbone. This infrastructure network can be connected to the Internet within a gateway. As we are interested in VANET for highways, each AP is situated along the road and covers a partial highway area. The figure 2 shows an example of such an architecture. The link between the APs is fixed and can be wireless or wired. 


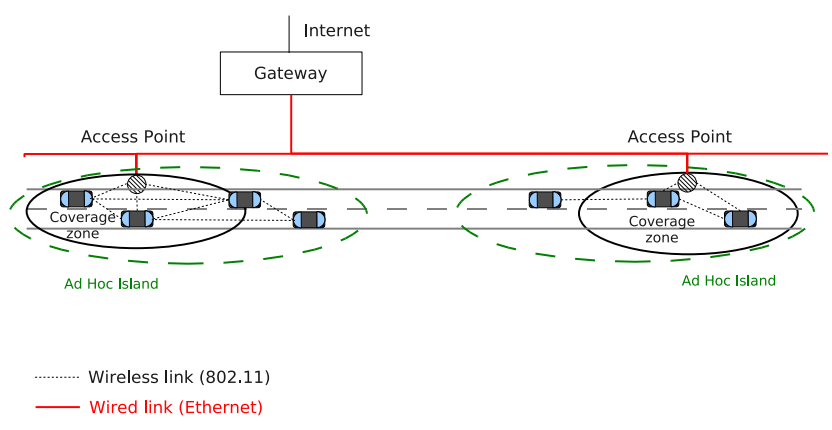

Fig. 2. Our architecture : highway with access points

Each node in an ad hoc island can access to an AP which is connected to all the other APs.

This architecture can be viewed as a particular Mesh system where the routers or APs are connected together within an unidimensional graph. To overcome the mobility problems, we use the same principle as in Mesh network, based on a single routing protocol both for the fixed part and for the wireless mobile one. It means that when a node is moving, the routing paths are updated, instead of using a mobility management protocol. The path from the nodes to the AP can be indirect, i.e., having several nodes like in an ad hoc network (multihop path). The introduction of APs in VANET introduces new problems to deal with.

\section{Introducing Mechanims of VHANET}

In this part, we are introducing the main features of a hybrid ad hoc network. Here, we use also a double interface node (the AP) to connect ad hoc nodes on one hand and the other APs on the other hand; nodes and AP being managed in the same way.

One of the first extensions we provide to hybrid ad hoc network consists of the nearest AP discovery process [22]; the nearest AP being reached using one or several hops. Conversely, the APs must know the path to each accessible node; this is performed using a node registration process. As an AP manages an ad hoc island using node registration, when the node goes to another ad hoc island, the mobile node has to execute handover from initial island to the other.

AP Discovery: Our main objective is to design a system which is scalable and which improves the network connectivity. In our mesh architecture, fast mobile nodes will quickly change of AP attachment and have to learn the path to their new AP as fast as possible. Instead of using normal routing process to access the nearest $\mathrm{AP}$, we provide an $\mathrm{AP}$ discovery process. AP discovery can be performed using AP advertisement messages periodically broadcasted in clusters. Thus, a node maintains a list of accessible APs, selects the best $\mathrm{AP}$ and sends to it a registration request. If the path to the AP is broken or a better AP is discovered, the node must change its AP and initiates a handover. As for Gateway
Discovery [23], AP discovery can be performed either by a reactive or by a proactive process :

- Reactive discovery: each time a node wants to access the AP, it broadcasts a message throughout the MANET soliciting a connection to the AP.

- Proactive discovery: every APs periodically broadcast an AP advertisement throughout their ad hoc island.

Handover Management: In our hybrid ad hoc network, the handover is driven by terminals because they are registering to the new AP. However, choosing the best AP is difficult because each candidate AP can be accessed by several paths. The nodes maintain a list of paths to all reachable APs. This list is updated when a new AP advertisement is received.

In cellular systems, the nodes trigger handover whenever the signal becomes too low to reach their current base station. It allows to react before the route failure. In VHANET, mobile nodes can not sense the radio channel of the AP because the path to AP can go through several nodes. As a consequence, the connection failure is detected on receiving the RouteError routing message when the node attempts to send a packet to an AP. In this case, the node has to register again to some AP or to the best AP in the list. Thus, in hybrid ad hoc network, a mobile node does not identify an AP by it's ID (IP address, MAC address or other ID), but it is identified with the path to the AP. The node's AP entry key is then a path to the AP.

TTL value of Route or Position Request: In reactive ad hoc protocols, the route discovery is performed using a route request message sent by the source. In geographic ad hoc protocols, the position of destination is known using a location request. The resulted path or position can be sent by a node in the same island or indirectly through the current AP. In the latter case, the AP sends the reply message instead of the destination or location server. A node is directly reachable if the hop count to this node is lower than the TTL value of request message. Whereas in DSR, the value of TTL parameter in RREQ messages is fixed to a default value, in VHANET, we are adjusting the TTL of AP advertisement message depending on the density of APs in the backbone. This feature allows the protocol to be scalable to the network size.

We have simulated these extensions added to a reactive protocol, DSR, to compare performance criteria of a flat network with those of a basic reactive protocol.

\section{Simulation Scenarios AND Numerical Results}

A vehicular traffic simulator have been developed to represent faithfully the traffic of vehicles. In the literature, several simulators have been designed for the simulation of a street context where others are dedicated to represent highway or grid city model [24], [25], [26], [27], [28], [29]. As, we consider a unidirectional highway, we have developed our own vehicular traffic simulator to represent node movements in a realistic way. It is based on a micromobility model [30] and consists for each vehicle in emulating the individual driver behaviors. On a highway, the driver is confined to accelerate, brake or change lanes. We assume that there is no on-ramp on our section of highway. A desired speed is associated to each 


\begin{tabular}{|r|l|}
\hline ApAdv Hello interval & $500 \mathrm{~ms}$ \\
\hline APAdv interval & $5000 \mathrm{~ms}$ \\
\hline APEntry timeout & $7500 \mathrm{~ms}$ \\
\hline Transport layer & $\mathrm{UDP}$ \\
\hline Bitrate & $10 \mathrm{~kb} / \mathrm{s} \mathrm{CBR}$ \\
\hline Wireless Bandwith & $11 \mathrm{Mb} / \mathrm{s}$ \\
\hline Wired Bandwith & $100 \mathrm{Mb} / \mathrm{s}$ \\
\hline Radio range & $250 \mathrm{~m}$ \\
\hline Simulation time & $90 \mathrm{~s}$ \\
\hline
\end{tabular}

TABLE I

FIXED SIMULATION PARAMETERS

vehicle which corresponds to the speed that the driver would have reached if he had been alone on its lane. If the driver is alone, he adapts its acceleration to reach its desired speed (free flow regime). If it is not alone, he adapts its acceleration to the vehicle just in front of him (car following regime). He can also change lanes if the other lane traffic conditions seem better. All these decisions are function of the environment of the vehicles (speed and distance) and random variables are used to introduce a different behavior for each car. Our simulator does not generate trace, but is a module for Jist/Swans[31], the network simulator we use for protocols simulation. We use the IEEE 802.11 MAC interface module of Swans and we have developped the code for DSR extended protocol.

\section{A. Simulation scenarios}

To show the improvements of our solution and its properties, we have considered and compared three architectures: original DSR protocol in a flat network, hybrid ad hoc network either with proactive AP discovery or with reactive AP discovery. In order to evaluate the scalability of a hybrid ad hoc protocol, the overhead, delay and throughput criteria have been analyzed under several road lengths. Basic scenario consists of vehicles driving on a highway whereas traffic and communication scenarios are the following: several pairs of nodes communicate with each other. Only one pair of nodes communicates per kilometer; if the road length is $20 \mathrm{~km}$ long, 20 pairs of nodes are communicating at the same time. Several parameters have been determined in the first calibration phase for best performance which are then fixed for later simulations. See table I. The default traffic density value is fixed to 8 vehicles per $\mathrm{km}$ per lane. In order to evaluate the behaviour of our solution and its scalability propertie, we vary several parameters which are involved in load: number of APs, length of road, number of communicating nodes pairs and traffic density per $\mathrm{km}$.

\section{B. Numerical Results}

Overhead : The overhead is defined as the number of signaling packets (used for advertisement, discovery and registration) over the total number of sent packets. We have estimated the overhead depending on the road length (Fig. 3) and the traffic density (Fig. 6). As a consequence of its lack of scalability, in a flat network overhead grows linearly while

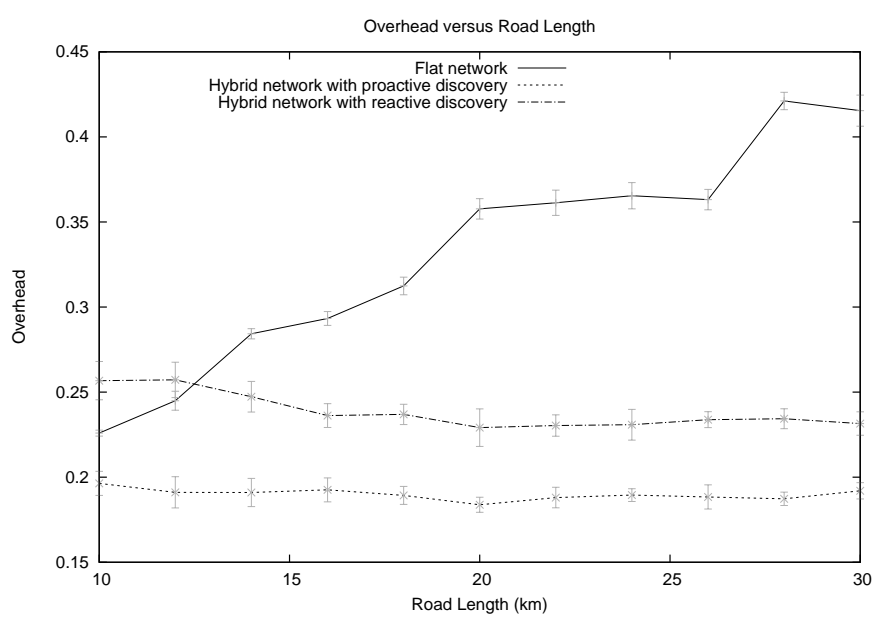

Fig. 3. Overhead versus Road Length

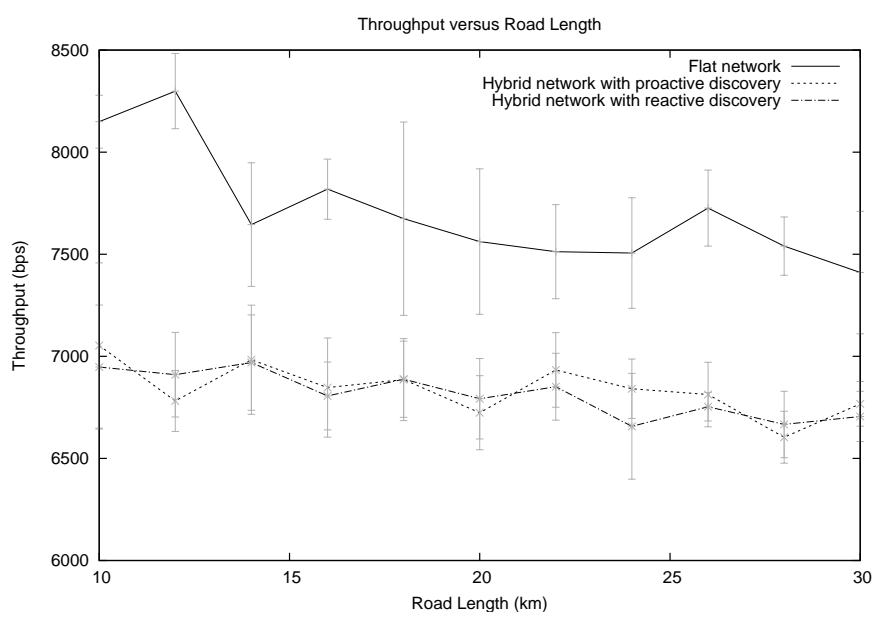

Fig. 4. Throughput versus Road Length

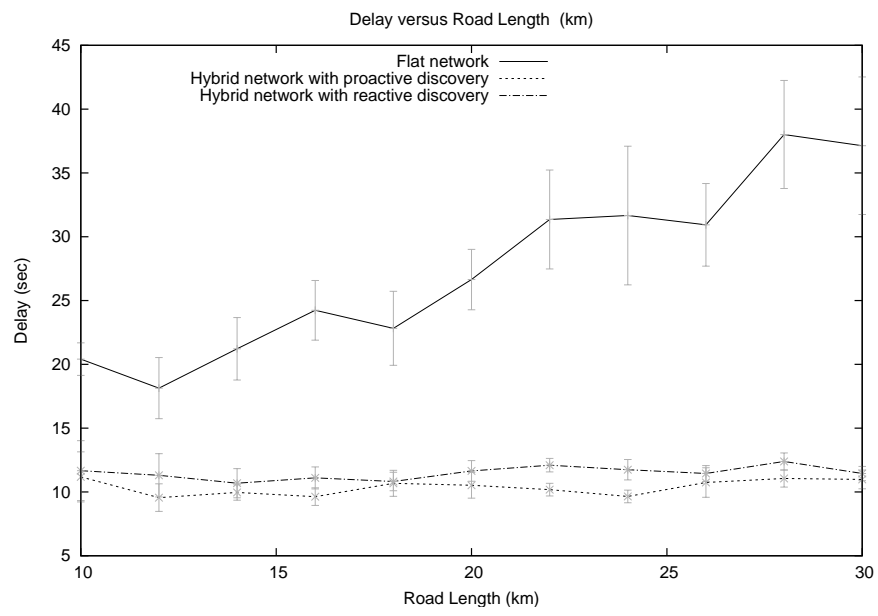

Fig. 5. Delay versus Road Length 


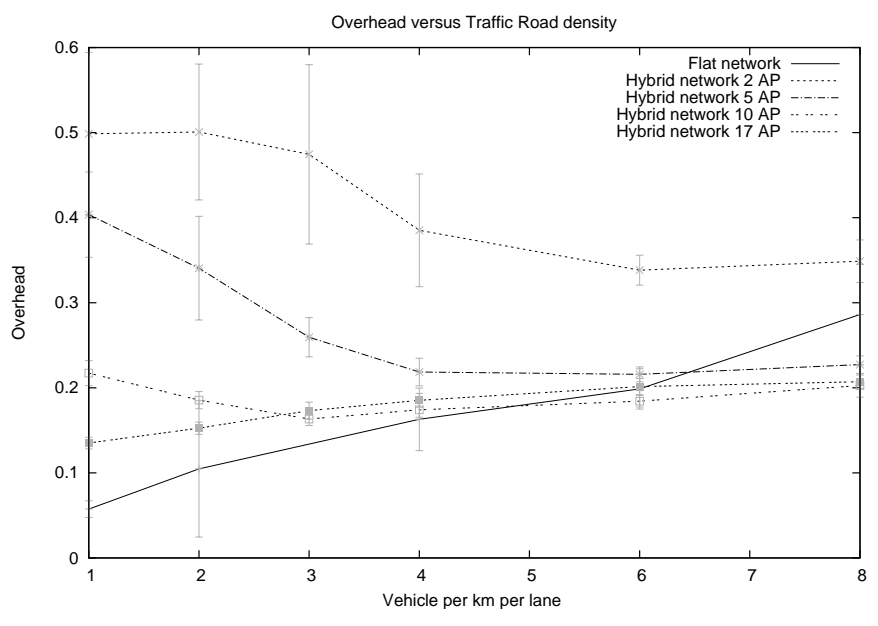

Fig. 6. Overhead versus Traffic Road density

in a hybrid ad hoc network, overhead is quite constant (Fig. 3 ) or is decreasing (Fig. 6).

Delay: The delay consists of the elapsed time between the request sending and its received response. For the flat network, the delay increaser linearily with the network size (Fig. 5), whereas in VHANET, the delays have the same behavior for both proactive and reactive AP discovery protocols.

Throughput : In contrast, the throughput is better for a flat network than for the VHANET (Fig. 4). Indeed, when a vehicle changes of AP, the link is broken in the VHANET. In this case, the delay takes into account the failure detection time (RouteError receiving) and the re-register delay. This delay is called a handover blackout time.

During this handover execution, the data packets are lost, so the global throughput is altered. Micro-mobility protocols provide some methods to avoid handoff packet losses, as in [32], [33], [34], [35]. A link break prediction and a forwarding of the undelivered packets to the new AP allow to reduce the number of lost packets. A similar method would achieve a better throughput in our protocol.

Scalability result : A network protocol is scalable if the overhead does not increase with the network size. In a flat network, the route request or the position request are broadcasted to the whole network. Thus in a highway network, as the forwarder nodes number, the overhead increase linearly regards to the network size. In a hybrid network, the AP provides a server for the location or path information to a node. The request is not sent to all the network, but just in its ad hoc island. With a good number of APs, the overhead can be minimized. In next section, we are studying the optimal number of APs

Connectivity Improvement: To show the connectivity improvement and the optimal access point density, overhead and delay are evaluated function of road traffic density, i.e. the number of vehicles per $\mathrm{km}$ and per lane. We use several sets of access points. The road length is fixed to $10 \mathrm{~km}$, and full coverage is reached with 20 APs. Here, we use proactive

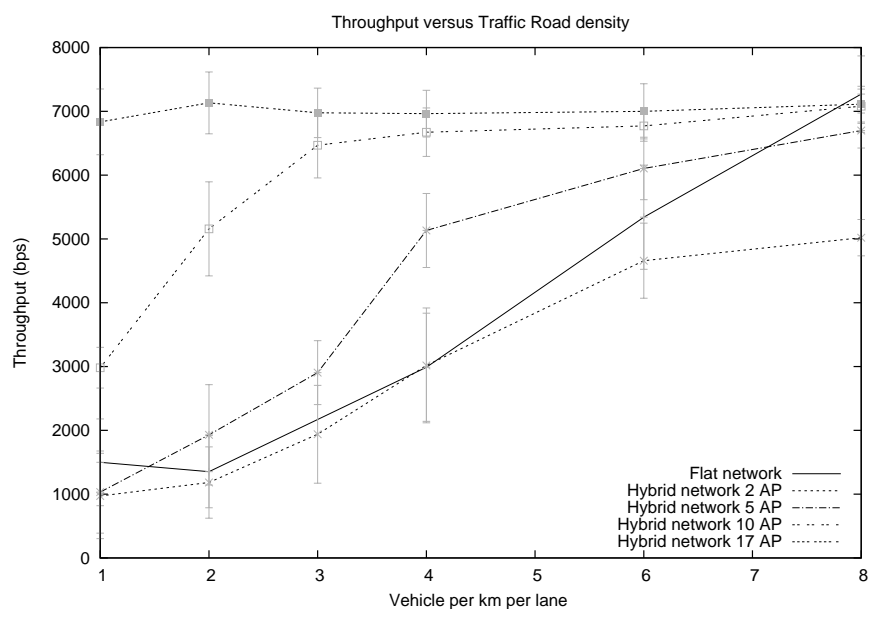

Fig. 7. Throughput versus Traffic Road density

AP discovery. In this simulation, DSR protocol is evaluated with a flat network to show the advantages of hybrid ad hoc architecture. The overhead is low with a flat network for low vehicle densities, see figure 6 , and increases for higher density. Consequently, we obtain the best performance for the throughput. As exposed in figure 7, when the infrastructure is composed of more than $5 \mathrm{APs}$, the throughput is always higher for a hybrid network than for a flat network. For small vehicle densities, the compromised value is 10 APs i.e. one AP per $\mathrm{km}$. Above 8 vehicles per $\mathrm{km}$ and per lane, a hybrid ad hoc network does not improve the performance due to overhead increase.

Finally, the results show that the overhead is improved with an optimal number of APs and the overhead is lower with a hybrid network than with a flat network.

\section{CONCLUSION}

In this paper, we have introduced a Vehicular Hybrid Ad hoc Network similar to a mesh network architecture. The network is composed of two types of node: the APs and the mobile node. In VHANET, APs are connected together using a fixed wired infrastructure network along the highway. The mobile node communicate among each other in an ad-hoc manner or through the APs network. One motivation of this hybrid architecture is the poor connectivity of Vehicular Ad Hoc network due to the vehicles traffic density and to the high speed of vehicles. As in a Mesh network, VHANET does not use mobility management protocol but uses an Ad hoc routing protocol. Due to the complexity of proactive routing protocols, influenced by the number of nodes and edges, these protocols do not operate well in a highly dynamic MANET. Thus, for VHANET, we are interested in both geographic and reactive routing protocols which operate well with high mobility and process similarily their requests either to find the route to destination or the destination position. Unfortunately, in a highway network, both methods suffer of the same drawback: its lack of scalability. This drawback generally leads to limit 
the network size. Our first motivation was then to improve the scalability of protocols for VHANET. We choose to extend DSR routing protocol with new capabilities: AP discovery, AP registration and Handover management, adjusted TTL value for RouteRequest. Using traffic and protocol simulations, we compare our VHANET solution to a flat network in terms of overhead, delay and throughput criteria under several values of road lengths or traffic densities. Our results exhibit that the overhead is not sensitive to network size increase in contrast with the flat network. Moreover, performance are better for the VHANET than for a flat network, except for throughput because in VHANET, the handovers are not seamless. We have also searched the optimal value of the number of APs and given some clues. This study will be further continued using a more formal analysis. Our further work will consist in comparing extending DSR to a geographic routing protocol.

\section{REFERENCES}

[1] F. Li and Y. Wang. Routing in vehicular ad hoc networks: A survey. Vehicular Technology Magazine, IEEE, 2(2):12-22, 2007.

[2] Holger Füßler, Martin Mauve, Hannes Hartenstein, Michael Käsemann, and Dieter Vollmer. A Comparison of Routing Strategies for Vehicular Ad Hoc Networks. Technical Report TR-02-003, Department of Computer Science, University of Mannheim, July 2002.

[3] David B. Johnson, David A. Maltz, and Josh Broch. Dynamic source routing in ad hoc network. In Mobile Computing, Kluwer Academic Publishers, pages 153-181, 1996.

[4] Muriel Mabiala, Anthony Busson, and Véronique Vèque. Inside vanet: Hybrid network dimensioning and routing protocol comparison. In VTC Spring, pages 227-232, 2007.

[5] SM Das, H. Pucha, and YC Hu. Performance comparison of scalable location services for geographic ad hoc routing. INFOCOM 2005. 24th Annual Joint Conference of the IEEE Computer and Communications Societies. Proceedings IEEE, 2, 2005.

[6] Y. Ko and N. H. Vaidya. Location-Aided Routing (LAR) in Mobile Ad Hoc Networks. In ACM MOBICOM 1998, Octobre 1998

[7] S. Basagni, I. Chlamtac, V.R. Syrotiuk, and B.A. Woodward. A distance routing effect algorithm for mobility (DREAM). Proceedings of the 4th annual ACM/IEEE international conference on Mobile computing and networking, pages 76-84, 1998.

[8] Jinyang Li, John Jannotti, Douglas S. J. De Couto, David R. Karger, and Robert Morris. A scalable location service for geographic ad hoc routing. In MobiCom '00: Proceedings of the 6th annual international conference on Mobile computing and networking, pages 120-130, New York, NY, USA, 2000. ACM.

[9] Holget Füßler, Jörg Widmer, Michael Käsemann, Martin Mauve, and Hannes Hartenstein. Contention-based forwarding for mobile ad-hoc networks. In Elsevier's Ad Hoc Networks, volume 1, 2003.

[10] Josh Broch, David A. Maltz, David B. Johnson, Yih chun Hu, and Jorjeta Jetcheva. A performance comparison of multi-hop wireless ad hoc network routing protocols. In In Proc. Mobicom, pages 85-97, 1998.

[11] Vincent D. Park and M. Scott Corson. A performance comparison of the temporally-ordered routing algorithm and ideal link-state routing. In In Proceedings of IEEE International Symposium on Systems and Communications. IEEE Computer, pages 592-598. Society Press, 1998.

[12] SR Das, CE Perkins, and EM Royer. Performance comparison of two on-demand routing protocols for adhoc networks. INFOCOM 2000. Nineteenth Annual Joint Conference of the IEEE Computer and Communications Societies. Proceedings. IEEE, 1, 2000.

[13] César A. Santiváñez, Bruce Mcdonald, Ioannis Stavrakakis, and Ram Ramanathan. On the scalability of ad hoc routing protocols. In INFOCOM 2002. Twenty-First Annual Joint Conference of the IEEE Computer and Communications Societies. Proceedings. IEEE, volume 3, pages 1688-1697 vol.3, 2002.

[14] B.J. Kwak, N.O. Song, and L.E. Miller. On the Scalability of Ad Hoc Networks: a traffic analysis at the center of a network. IEEE WCNC 2004.
[15] A. Hamidian. AODV+: An extension to AODV, Source code for implementation on $\mathrm{ns} 2[\mathrm{DB} / \mathrm{OL}]$.

[16] C. E. Perkins and E. M. ROyer. Ad hoc on-demand distance vector routing. In IEEE Workshop on Mobile Computing Systems and Applications 1999, pages 90-100, Fevrier 1999.

[17] NS2 : the network simulator. http://www.isi.edu/nsnam/ns.

[18] Ryuji Wakikawa and Charles E. Perkins and Anders Nilsson and Antti J. Tuominen. Global connectivity for IPv6 mobile ad hoc networks. Work in Progress, March 2006.

[19] J. Broch, D.A. Maltz, and D.B. Johnson. Supporting Hierarchy and Heterogeneous Interfaces in Multi-Hop Wireless Ad Hoc Networks. Workshop on Mobile Computing at I-SPAN, 1999.

[20] I. Chakeres and C. Perkins. Dynamic MANET On-demand (DYMO) Routing. Work in Progress, March 2007.

[21] M. Bechler, L. Wolf, O. Storz, and WJ Franz. Efficient discovery of Internet gateways in future vehicular communication systems. Vehicular Technology Conference, 2003. VTC 2003-Spring. The 57th IEEE Semiannual, 2, 2003.

[22] P. Ratanchandani and R. Kravets. A hybrid approach to Internet connectivity for mobile ad hoc networks. Wireless Communications and Networking, 2003. WCNC 2003. 2003 IEEE, 3, 2003.

[23] M. Ghassemian, P. Hofmann, C. Prehofer, V. Friderikos, and H. Aghvami. Performance analysis of Internet gateway discovery protocols in ad hoc networks. Wireless Communications and Networking Conference, 2004. WCNC. 2004 IEEE, 1, 2004.

[24] MIT inteligent transportation systems. http://mit.edu/its/mitsimlab.html.

[25] CORSIM microscopic traffic simulation model http://mctrans.ce.ufl.edu/featured/tsis/version5/corsim.htm.

[26] W. Kronjäger and D. Hermann. Travel time estimation on the base of microscopic traffic flow simulation. ITS World Congress, 1999.

[27] K.J. Wong, B.S. Lee, B.C. Seet, G. Liu, and L. Zhu. BUSNet: Model and Usage of Regular Traffic Patterns in Mobile Ad Hoc Networks for Inter-Vehicular Communications. Proc. ICT, 2003.

[28] A. Kamat and R. Prakash. Effects of link stability and directionality of motion on routingalgorithms in MANETs. Computer Communications and Networks, 2000. Proceedings. Ninth International Conference on, pages $380-385,2000$.

[29] F. Karnadi, Z. Mo, and K. Lan. Rapid Generation of Realistic Mobility Models for VANET. International Conference on Mobile Computing and Networking (ACM MOBICOMM 2005), ACM Press, New York, 2005.

[30] Kazi I. Ahmed. Modeling Drivers'Acceleration and Lane Changing Behavior. Doctor of science in transportation systems, Massachusetts Institute of Technology, Massachusetts Institute of Technology, Boston (MA), 1999.

[31] Jist / Swans : Java in simulation time / scalable wireless ad hoc network simulator. http://jist.ece.cornell.edu.

[32] AT Campbell, J. Gomez, and AG Valko. An overview of cellular IP. Wireless Communications and Networking Conference, 1999. WCNC. 1999 IEEE, pages 606-610, 1999.

[33] Ramachandran Ramjee, Thomas F. La Porta, S. Thuel, Kannan Varadhan, and S. Y. Wang. HAWAII: A domain-based approach for supporting mobility in wide-area wireless networks. In ICNP, pages 283-292, 1999.

[34] H. Soliman, C. Castelluccia, K. El Malki, and L. Bellier. Hierarchical mobile IPv6 mobility management (HMIPv6). RFC 4140 (Experimental), August 2005.

[35] R. Koodli. Fast handovers for mobile IPv6. RFC 4068 (Experimental), July 2005 . 